\title{
Short Acquisition Time PET/MR Pharmacokinetic Modelling using CNNs
}

\author{
Catherine $\mathrm{J} \mathrm{Scott}^{1}$, Jieqing Jiao ${ }^{1}$, M. Jorge Cardoso ${ }^{2,1}$, Kerstin Kläser $^{1}$, \\ Andrew Melbourne ${ }^{2,3}$, Pawel J Markiewicz ${ }^{1}$, Jonathan M Schott ${ }^{4}$, Brian F \\ Hutton $^{5}$, and Sébastien Ourselin ${ }^{2}$ \\ ${ }^{1}$ Centre for Medical Image Computing, University College London, London, UK \\ ${ }^{2}$ School of Biomedical Engineering and Imaging Sciences, King's College London \\ ${ }^{3}$ Dept. of Medical Physics and Biomedical Engineering, University College London \\ ${ }^{4}$ Dementia Research Centre, Institute of Neurology, University College London, UK \\ ${ }^{5}$ Institute of Nuclear Medicine, University College London, London, UK
}

\begin{abstract}
Standard quantification of Positron Emission Tomography (PET) data requires a long acquisition time to enable pharmacokinetic (PK) model fitting, however blood flow information from Arterial Spin Labelling (ASL) Magnetic Resonance Imaging (MRI) can be combined with simultaneous dynamic PET data to reduce the acquisition time. Due the difficulty of fitting a PK model to noisy PET data with limited time points, such 'fixed- $R_{1}$ ' techniques are constrained to a $30 \mathrm{~min}$ minimum acquisition, which is intolerable for many patients. In this work we apply a deep convolutional neural network (CNN) approach to combine the PET and MRI data. This permits shorter acquisition times as it avoids the noise sensitive voxelwise PK modelling and facilitates the full modelling of the relationship between blood flow and the dynamic PET data. This method is compared to three fixed- $R_{1}$ PK methods, and the clinically used standardised uptake value ratio (SUVR), using $60 \mathrm{~min}$ dynamic PET PK modelling as the gold standard. Testing on 11 subjects participating in a study of pre-clinical Alzheimer's Disease showed that, for 30 min acquisitions, all methods which combine the PET and MRI data have comparable performance, however at shorter acquisition times the CNN approach has a significantly lower mean square error (MSE) compared to fixed- $R_{1} \mathrm{PK}$ modelling $(p=0.001)$. For both acquisition windows, SUVR had a significantly higher MSE than the CNN method $(p \leq 0.003)$. This demonstrates that combining simultaneous PET and MRI data using a CNN can result in robust PET quantification within a scan time which is tolerable to patients with dementia.
\end{abstract}

\section{Introduction}

For the accurate quantification of tracer target density $\left(B P_{N D}\right)$, such as amyloid$\beta$ burden in Alzheimer's disease, phamacokinetic (PK) modelling of dynamic Positron Emission Tomography (PET) data requires the acquisition to cover the delivery, binding and washout of the injected radiotracer. This may take 60 mins 
or more which is not clinically feasible, due to patient discomfort, scanner availability, and increased motion. A framework which reduces the PET acquisition time by incorporating simultaneously acquired arterial spin labelled (ASL) MRI data into the PK model has been proposed [1]. This involves three steps; conversion of ASL cerebral blood flow (CBF) maps into the relative PET tracer delivery parameter $\left(R_{1}\right)$, extrapolation of the PET input function $\left(C_{R}\right)$ to account for the missing time-points, and PK model fitting to the measured PET data using fixed $R_{1}$ and extrapolated $C_{R}$. We refer to this as the 'fixed $R_{1}$ method'. Unlike the clinically used standardised uptake value ratio (SUVR), this method can account for changes in blood flow, which can confound estimates of target density in longitudinal studies [2]. However, due the difficulty of fitting a PK model to noisy PET data with limited time points, this technique is constrained to a 30 min minimum acquisition time, which may still be intolerable for some patients.

The 'fixed- $R_{1}$ ' approach estimates $R_{1}$ from ASL-CBF independently from dynamic PET fitting for target density $\left(B P_{N D}\right)$ and washout rate $\left(k_{2}\right)$. This implementation cannot explicitly model the known influence of CBF on washout, due to high uncertainty in washout estimation, and the complex relationship which is dependent on the local tissue tracer kinetics [3]. Furthermore, the extrapolation of the input function, $C_{R}$, uses scaled population data under the assumption that tracer washout in this region is equal to the average population value. This assumption is violated in the case of disease or blood flow changes.

In this work we propose a deep learning (DL) framework to achieve PET quantification for a short acquisition time in a single step. We avoid the noise sensitive voxelwise PK curve fitting step, through the use of deep convolutional neural networks which enforce spatial regularisation across the receptive field. Our approach negates the need for explicit modelling between CBF, tracer delivery and tracer washout, as these relationships are learnt from the data and modelled in conjunction with the dynamic PET data. This approach also avoids $C_{R}$ extrapolation, overcoming the limitation of a population tracer washout rate.

To our knowledge, this is the first time in which DL has been applied to PET PK modelling. This is due to the availability of robust models to describe standard data and the lack of one-to-one mapping between model parameters and dynamic PET data. However, the standard models are not sufficient to describe PET data with missing time-points. Furthermore, the incorporation of ASL-CBF constrains the parameter estimation. DL was chosen for its ability to model the underlying relationship between ASL-CBF and the delivery, binding and washout of the PET tracer without explicit feature extraction. By exploiting all of the PET and MRI information, and avoiding voxelwise fitting, this framework provides more robust estimates of target density with a shorter acquisition.

\section{Methods}

\subsection{Deep Learning Framework for $B P_{N D}$ Estimation}

The framework performs regression of PET target density $\left(B P_{N D}\right)$ from PET and MRI data directly. The network was implemented in NiftyNet [4] using 
the 'highresnet' convolutional neural network with 20 convolutional layers [5], which uses a stack of residual dilated convolutions with increasingly large dilation factors. For training we used adaptive moment estimation (Adam) with an initial learning rate of $10^{-3}$, and a root mean square error loss function. The networks were initialised randomly and trained for a maximum of 50,000 iterations. The training patch size was 56x56x56 voxels and a smoothed brain mask was used for adaptive sampling. Random rotation and scaling transformations of $\pm 10 \%$ were used for training data augmentation. All inputs were 3D image volumes: the ASL-CBF maps, the structural T1 weighted MRI, and the dynamic PET data, which were entered as one frame per channel, see fig. 1.

\subsection{Gold Standard PK Modelling}

The linearised simplified reference tissue model (SRTM) is used for gold standard PET quantification (1). Basis functions for $C_{R}(t) \otimes e^{-\theta t}$ are pre-calculated over a physiologically plausible range of $\theta[6]$, where $C_{R}(t)$ is the tracer concentration in the reference region. $C_{R}(t)$ is used as an input function since the reference region, cerebellar grey matter, is considered to be devoid of the imaging target. $C_{T}(t)$ is the measured tracer concentration in the target tissue. The model parameters are: $R_{1}$ (the delivery rate constant in the target tissue relative to reference tissue), $k_{2}$ (the transfer rate constant from target tissue to blood), and the parameter of interest $B P_{N D}$ (the binding potential which is related to target density and consequently amyloid- $\beta$ burden). The parameters are estimated via curve fitting to $C_{R}(t)$ and $C_{T}(t)$ acquired over $t=0: 60$ mins.

$$
\begin{array}{r}
C_{T}(t)=R_{1} C_{R}(t)+\phi C_{R}(t) \otimes e^{-\theta t} \\
\text { where } \phi=k_{2}-R_{1} k_{2} /\left(1+B P_{N D}\right), \quad \theta=k_{2} /\left(1+B P_{N D}\right)
\end{array}
$$

\subsection{Acquisition window definition}

For gold standard PK modelling the scan starts at tracer injection, $t_{s}=0$, with a duration of $t_{d}=60$ mins. However, for the short acquisition methods $t_{s}>0$, and $t_{d}$ is chosen to fit clinical requirements. We optimise the timing window, $t=t_{s}: t_{s}+t_{d}$, for each method at different $t_{d}$ 's. This was performed over $t=30: 60$ mins, as this period is recommended for routine clinical scans using this tracer.

\subsection{Comparison Methods for Short Acquisition PET Quantification}

We compare the proposed technique to four short PET acquisition methods: three fixed- $R_{1}$ methods, and the clinical standard, SUVR.

Fixed $\boldsymbol{R}_{\mathbf{1}}$ methods. Two methods are used to derive $R_{1}$ from ASL-CBF: the linear regression (LR) method [1], and the image fusion (IF) method [7]. Both methods require a database of subjects with 60 mins of PET data and ASL. 
The LR method performs linear regression between $R_{1}$ and ASL-CBF on the database and the relationship is applied to an unseen ASL-CBF map. For IF, the local similarity between the unseen ASL-CBF map and those in the database is used to weight the propagation of $R_{1}$ database values into the subject's space. An additional method using the gold standard $R_{1}$ (true $R_{1}$ ) is also included to demonstrate the upper limit of this approach, where $R_{1}$ is estimated perfectly from the ASL data.

For all three methods the estimation of $B P_{N D}$ was carried out as previously described $[1,7]$. Briefly, the reference region, $C_{R}$ is

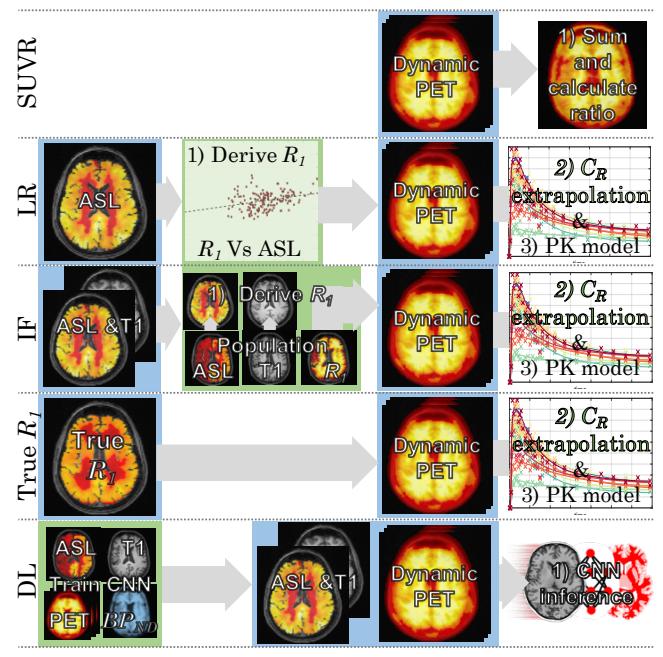

Fig. 1: Overview of methods tested. Blue boxes indicate input subject data and green boxes population data. extrapolated to $t=0$, at tracer injection, by scaling the mean population $C_{R}$ to the measured data using a linear least squares fit, then the derived $R_{1}$ value and the extrapolated $C_{R}$ are used in (1) to estimate $k_{2}$ and $B P_{N D}$ from the measured PET data.

Standardised uptake value ratio (SUVR). SUVR is calculated by dividing the image $\left(C_{T}\right)$ by the mean value in the reference region $\left(C_{R}\right)$ to yield relative tracer uptake, which can not take blood flow into account. For comparison with $B P_{N D}$, one is subtracted, as $B P_{N D} \approx \frac{C_{T}}{C_{R}}-1$. SUVR is calculated over different timing windows by first summing the relevant reconstructed frames.

\subsection{Data Acquisition and Pre-processing}

Database construction. For each subject the T1 and ASL-CBF MR images were affinely registered into PET space. The subjects were randomly split between training $(38, \sim 70 \%)$, validation $(6, \sim 10 \%)$ and testing $(11, \sim 20 \%)$. The input data used and an overview of each methodology is summarised in fig. 1, where the dynamic PET data include the frames acquired over $t=t_{s}: t_{s}+t_{d}$.

PET data. 60 mins of PET data were acquired following intravenous injection of an amyloid- $\beta$ targeting radiotracer, $\left[{ }^{18} \mathrm{~F}\right]$ florbetapir. Dynamic PET data were binned into $15 s \times 4,30 s \times 8,60 s \times 9,180 s \times 2,300 s \times 8$ time frames, such that all frames for $t \geq 20$ mins were 5 mins long. The data were reconstructed into $2 \times 2 \times 2 \mathrm{~mm}$ voxels, accounting for dead-time, attenuation (using synthetic CT), scatter, randoms and normalisation [8]. 


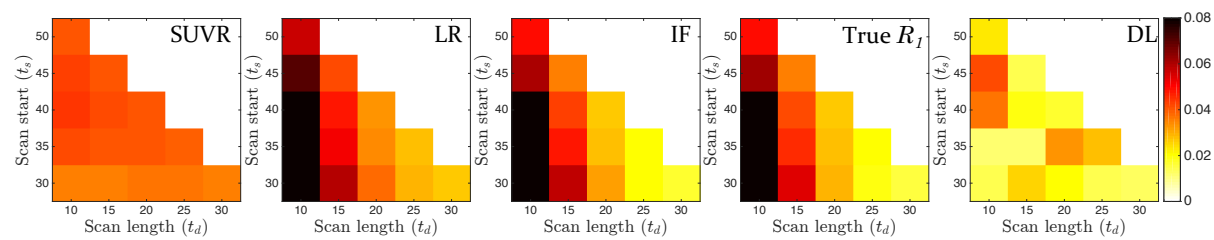

Fig. 2: MSE averaged across subjects for different timing windows and methods

ASL data. Pseudo-continuous ASL data were acquired at $t=55: 60$ mins, using a $3 \mathrm{D}$ GRASE readout at $3.75 \times 3.75 \times 4 \mathrm{~mm}$ and reconstructed to $1.88 \times 1.88 \times$ $4 \mathrm{~mm}$ voxels. 10 control-label pairs were acquired with a pulse duration and post labelling delay of $1800 \mathrm{~ms}$. Proton density, $S_{0}$, was estimated by fitting saturation recovery images, at three recovery times $(1,2,4 \mathrm{~s})$, for $\left[\mathrm{T} 1, S_{0}\right]$. Cerebral blood flow $(\mathrm{CBF})$ maps were then estimated from the ASL and saturation recovery images [9]. The parameter values were $0.9 \mathrm{ml} / \mathrm{g}$ for the plasma/tissue partition coefficient, $1650 \mathrm{~ms}$ for blood $\mathrm{T} 1$, and 0.85 for labelling efficiency.

\section{Experiments and Results}

Data. Imaging data were collected from 55 cognitively normal subjects participating in Insight 46, a neuroimaging sub-study of the MRC National Survey of Health and Development [10], who underwent simultaneous PET and multimodal MRI on a Siemens Biograph mMR 3T PET/MRI scanner. 11 subjects were used for testing, with the remaining subjects used in the database for training and validation.

Validation. The proposed deep learning (DL) method was compared to three fixed- $R_{1}$ methods (LR, IF and true $R_{1}$ ), and SUVR. $B P_{N D}$ estimation accuracy was assessed using the mean square error: MSE $=1 / v \sum_{v}\left(I_{v}^{\text {est }}-I_{v}^{G S}\right)^{2}$, where $I$ is intensity, $v$ is the number of voxels, $G S$ is the gold standard and est is the estimate. Statistical tests were performed using the Wilcoxon signed rank test.

\subsection{Method Comparison Over Different Timing Windows}

Fig. 2 shows the average MSE across subjects for different data acquisition windows for each method. Here, SUVR shows minimal influence from acquisition timing and length due to the simplicity of the technique. However, since it is not able to account for tracer delivery or washout it has a consistently high MSE. For the three methods which perform kinetic modelling with a fixed $R_{1}$ there is a strong time dependence, with the error increasing greatly as the scan time is reduced. Consequently they outperform SUVR for 30 min acquisitions, but for scans of less than 20 mins, where the number of datapoints is $\leq 4$, they produce a higher error. This is due to the difficulty in fitting the PK model to a few noisy datapoints, and the increased uncertainty in the extrapolation of $C_{R}$. 

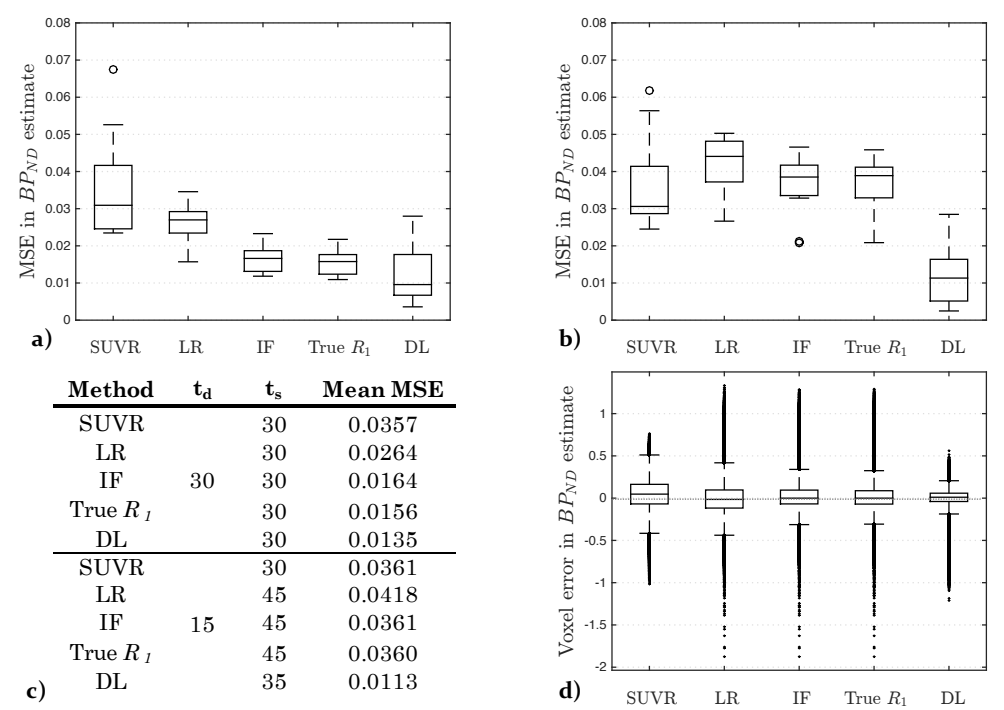

Fig. 3: Subject MSE for a) 30 and b) 15 min scans, summarised in c). The voxelwise error for all subjects for a 15 min scan is shown in d).

The deep learning based method (DL) shows a consistently low MSE across timing windows compared to the other techniques. This is because voxel based PK modelling, and $C_{R}$ extrapolation, which are acquisition length dependent, are avoided. Furthermore, blood flow information is leveraged to inform both tracer delivery and washout, reducing the acquisition time required relative to the fixed$R_{1}$ PK modelling techniques. For DL there is no clear trend to the acquisition window dependence which makes it more flexible for clinical implementation.

\subsection{Optimised Timing Window Method Comparison}

Based on the mean MSE, shown in fig. 2, the best timing window for each method was selected for a $30 \mathrm{~min}$ (6 frames) and $15 \mathrm{~min}$ (3 frames) scan, representing a long clinical scan and a tolerable scan duration respectively, see fig. 3c).

30 min optimised acquisition. Fig. 3a) shows the MSE across subjects for the $30 \mathrm{~min}$ acquisition window. As expected the fixed- $R_{1}$ methods have a lower MSE than SUVR due to the more accurate modelling of tracer delivery and washout. For this acquisition length the benefit of using the deep learning approach is limited compared to the fixed $R_{1}$ methods, and the difference in MSE did not reach statistical significance. However, DL has a significantly lower MSE compared to SUVR $(p=0.003)$. Fig. 4a) shows an example subject, which highlights the good performance of the IF, true $R_{1}$ and DL methods, while SUVR shows a large over estimation. The LR method shows corruption due to artefacts in the ASL-CBF map which propagate directly into the $B P_{N D}$ estimation. 
15 min optimised acquisition. When the scan time is reduced to 15 mins the MSE in the fixed- $R_{1}$ methods increases, even when using the true $R_{1}$ parameter. By contrast, the DL and SUVR methods maintain their performance levels. Now DL has a significantly lower MSE than both the fixed- $R_{1}$ methods $(p \leq 0.001)$ and SUVR $(p=0.001)$. The DL method also has a lower bias than all other methods, see fig. 3d), but this does not reach statistical significance.

Fig. 4b) shows the estimated $B P_{N D}$ images using a 15 min acquisition for the different methods for an example subject. Here, the noise in the fixed- $R_{1}$ methods is a result of the limited timepoints for the fit. SUVR gives a plausible estimate of $B P_{N D}$, however the image demonstrates a general overestimation of the target density compared to the true image. By contrast, the DL technique yields a low noise image due to the spatial regularisation inherent in the technique, with high accuracy as the model is able to combine the dynamic PET data with the blood flow information from the ASL to accurately estimate $B P_{N D}$.

\section{Discussion and Conclusion}

In this paper we present a deep learning approach to PET target density estimation, by combining dynamic PET data with MRI blood flow and structural images to significantly reduce the acquisition time to just 15 mins, compared to the gold standard 60 mins. This is applied to amyloid PET data which is used in the diagnosis and monitoring of Alzheimer's disease, as the symptoms of the disease necessitate short scans. This method was compared to the clinical standard, SUVR, as well as previously proposed techniques which fix the tracer delivery parameter $R_{1}$ using MRI blood flow data in the PET PK modelling to reduce the acquisition time. This demonstrated that, for a 30 min acquisition, the proposed technique performed comparably to the previously proposed fixed- $R_{1}$ techniques, and significantly better than SUVR $(p=0.003)$. When the acquisition window was reduced to 15 mins, the fixed- $R_{1}$ methods had insufficient data to fit the PK model. However, the deep learning method maintained its low MSE, which was significantly lower than the clinically used SUVR $(p=0.001)$.

This initial work proves the benefit of using deep learning to perform PET quantification where limited PET data means that the standard model fails. In the future we intend to build on this approach by explicitly encoding the PET frame timing information into the model. This would not only give the model more information, but also the potential to cope with discontinuous scans.

Acknowledgements This work was supported by the EPSRC UCL Centre for Doctoral Training in Medical Imaging (EP/L016478/1), UCL Leonard Wolfson Experimental Neurology Centre (PR/ylr/18575), MRC (MR/J01107X/1), EPSRC (NS/A000027/1), NIHR UCLH Biomedical Research Centre (inc. High Impact Initiative, BW.mn.BRC10269). Insight 1946 receives funding from Alzheimer's Research UK (ARUKPG2014-1946, ARUKPG2014-1946), MRC Dementia Platform UK (CSUB19166), The Wolfson Foundation, and Brain Research Trust. The Florbetapir tracer was kindly supplied by Avid Radiopharmaceuticals, a 


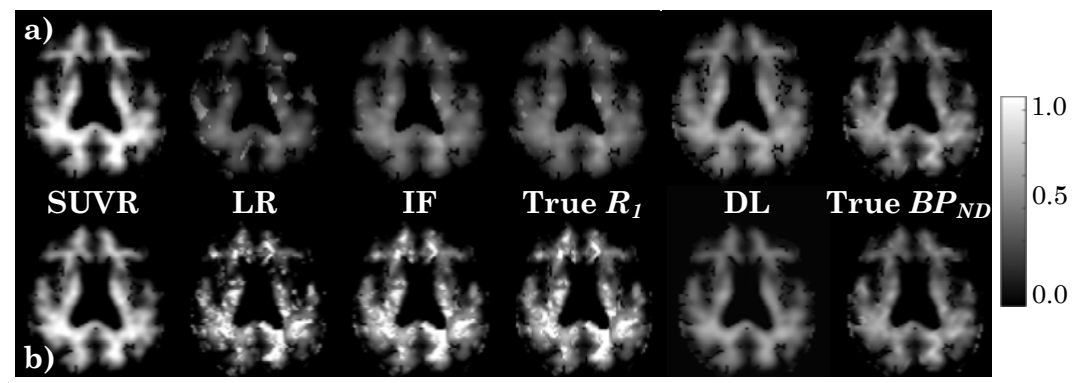

Fig. 4: Example subject $B P_{N D}$ maps optimised for a) 30 and b) 15 min acquisition, where the true $B P_{N D}$ is calculated over 60 mins for both.

wholly owned subsidiary of Eli Lilly. We are grateful to the Insight 46 participants for their involvement in this study and to K. Erlandsson for his advice.

\section{References}

1. Scott, C.J., Jiao, J., Melbourne, A., et al: ASL-incorporated Pharmacokinetic Modelling of PET Data With Reduced Acquisition Time: Application to Amyloid Imaging. Med Image Comput Comput Assist Interv, LNCS 9902 (2016) 406-413

2. van Berckel, B.N.M., Ossenkoppele, R., Tolboom, N., et al.: Longitudinal amyloid imaging using 11C-PiB: methodologic considerations. J Cereb Blood Flow Metab 54(9) (sep 2013) 1570-6

3. Wu, Y., Carson, R.E.: Noise reduction in the simplified reference tissue model for neuroreceptor functional imaging. J Cereb Blood Flow Metab 22(12) (2002) 1440-1452

4. Gibson, E., Li, W., Sudre, C., et al.: Niftynet: a deep-learning platform for medical imaging. In: arXiv preprint arXiv:1709.03485. (2017)

5. Li, W., Wang, G., Fidon, L., et al.: On the compactness, efficiency, and representation of 3D convolutional networks: Brain parcellation as a pretext task. In: Information Processing in Medical Imaging. (2017)

6. Gunn, R.N., Lammertsma, A.A., Hume, S.P., et al.: Parametric imaging of ligandreceptor binding in PET using a simplified reference region model. NeuroImage 6(4) (1997) 279-287

7. Scott, C.J., Jiao, J., Cardoso, M.J., et al.: Short Acquisition Time PET Quantification Using MRI-Based Pharmacokinetic Parameter Synthesis. Med Image Comput Comput Assist Interv, LNCS 10434 (2017) 737-744

8. Markiewicz, P.J., Ehrhardt, M.J., Erlandsson, K., et al.: NiftyPET: a Highthroughput Software Platform for High Quantitative Accuracy and Precision PET Imaging and Analysis. Neuroinformatics (2017) 1-21

9. Melbourne, A., Toussaint, N., Owen, D., et al: Niftyfit: a software package for multiparametric model-fitting of $4 \mathrm{D}$ magnetic resonance imaging data. Neuroinformatics 14(3) (2016) 319-337

10. Lane, C.A., Parker, T.D., Cash, D.M., et al.: Study protocol: Insight 46 a neuroscience sub-study of the MRC National Survey of Health and Development. BMC Neurology 17(1) (2017) 75 